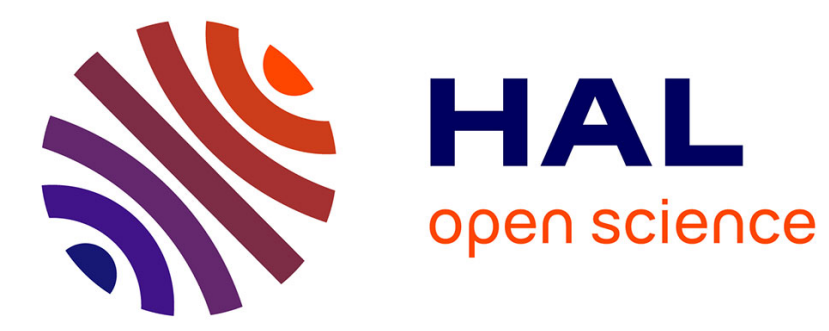

\title{
Cartes et autres objets variés et multiples. Un habitus littératien?
}

\author{
Jean-Marie Privat
}

\section{To cite this version:}

Jean-Marie Privat. Cartes et autres objets variés et multiples. Un habitus littératien?. Pratiques: linguistique, littérature, didactique, 2006, 131, pp.125-130. 10.3406/prati.2006.2122 . hal-03180249

\section{HAL Id: hal-03180249 \\ https://hal.univ-lorraine.fr/hal-03180249}

Submitted on 24 Mar 2021

HAL is a multi-disciplinary open access archive for the deposit and dissemination of scientific research documents, whether they are published or not. The documents may come from teaching and research institutions in France or abroad, or from public or private research centers.
L'archive ouverte pluridisciplinaire HAL, est destinée au dépôt et à la diffusion de documents scientifiques de niveau recherche, publiés ou non, émanant des établissements d'enseignement et de recherche français ou étrangers, des laboratoires publics ou privés. 


\section{Un habitus littératien?}

\section{Jean-Marie Privat}

\section{Résumé}

Cet article se propose de spécifier la structure du champ des pratiques littératiennes, d'établir la notion de coefficient discursif de littératie, enfin d'examiner la sémiotisation littératienne de notre « written world » et ses effets différenciés sur les usagers.

\section{Citer ce document / Cite this document :}

Privat Jean-Marie. Un habitus littératien ?. In: Pratiques : linguistique, littérature, didactique, $\mathrm{n}^{\circ} 131-132,2006 . \mathrm{pp} .125-$ 130 ;

doi : https://doi.org/10.3406/prati.2006.2122

https://www.persee.fr/doc/prati_0338-2389_2006_num_131_1_2122

Fichier pdf généré le 19/01/2021 


\title{
Un habitus littératien?
}

\author{
Jean-Marie Privat \\ Université Paul Verlaine - Metz \\ CELTED EA 3474
}

Loin de toute théorie romantique du « grand partage » entre cultures orales et cultures écrites ${ }^{(1)}$, l'étude des effets symboliques et pratiques des modes de communication sur nos modes de pensée et nos modes de vie implique un approfondissement du travail de spécification des modes d'existence de la littératie.

\section{Les trois formes du capital littératien}

La première clarification nécessaire concerne à mon sens les types de manifestation de la littératie. Il semble en effet heuristique d'identifier trois formes de capital littératien : le capital littératien objectivé, institutionnalisé et incorporé. Les dernières réflexions de J. Goody à propos des « technologies de l'intellect» ne s'inscrivent pas dans une autre perspective :

«Lorsque je parle de l'écriture en tant que technologie de l'intellect, je ne pense pas seulement aux plumes et au papier, aux stylets et aux tablettes, aussi complexes que soient ces instruments, mais aussi à la formation requise, l'acquisition de nouvelles compétences motrices, l'utilisation différente de la vue, ainsi qu'aux produits eux-mêmes, les livres qui sont rangés sur les étagères des bibliothèques, objets que l'on consulte et dans lesquels on apprend, et qu' on peut aussi, le moment venu, composer. » ${ }^{(2)}$

L'anthropologue et le didacticien de la culture écrite ne peuvent dès lors que réfléchir aux divers modes d'acquisition et d'articulation des différentes formes de ce type de capital culturel.

(1) J. Goody, « Retour au grand partage », La Raison graphique. La domestication de la pensée sauvage, J. Bazin et A. Bensa éds., Paris, Minuit, 1979, pp. 245-267. Sur une approche critique (linguistique, anthropologique et ethnologique) récente du « grand partage » à propos de la littératie, voir par exemple B. Street, "Introduction : the new literacy studies », Cross-cultural approaches to literacy, ed. by B. Street, Cambridge University Press, Cambridge, 1993, pp. 1-21. Voir aussi on line des contributions de chercheurs anglo-saxons en « New Literacy Studies".

(2) J. Goody, "Technologies on the Intellect: Writting and the Written Word », The Power of the Written Tradition, Smithsonian Institution Press, Washington and London, 2002, p. 133. 
L'état objectivé du capital littératien d'une société sinon d'un individu particulier se mesure par exemple aujourd'hui aux « 11.000 références de fournitures informatiques et de bureau, mobilier de bureau, articles scolaires et papeterie personnelle » du catalogue « Calipage 2006 / 626 pages » des distributeurs-conseils professionnels en objets marchands de la littératie...

L'état institutionnalisé de ce même capital se lit dans n'importe quelle bibliographie de lettré (ici un notable et érudit normand du XIX ${ }^{\mathrm{e}} \mathrm{s}$. en quête de toujours plus de légitimité savante) :

«Recherches archéologies, historiques, biographiques et littéraires sur La Normandie, par M. Louis Du Bois, Ancien Bibliothécaire de l'Ecole centrale de l'Orne ; Membres de plusieurs Académies de Paris, des Départements et de 1'Etranger ; Correspondant du Ministère de l'Instruction publique pour les travaux historiques [...], A Paris, Chez Dumoulin Libraire-Imprimeur, Quai des Augustins, $\mathrm{n}^{\circ} 13,1843 . »$

Enfin, l'état incorporé se réalise exemplairement dans la figure de l'homo litteratiens que Nietzsche diabolise comme "encyclopédie ambulante » et que Flaubert (sinon le professeur de lettres...) chérit comme idéale et fusionnelle bio-graphie :

« Je suis un homme-plume. Je sens par elle, à cause d'elle, par rapport à elle et beaucoup plus avec elle. » ${ }^{(3)}$

\section{Les coefficients de littératie}

La seconde manière d'analyser le concept de littératie pour le rendre opératoire consiste à dépasser la querelle idéologique et spéculative de l'opposition massive entre cultures orales et écrites, non pas seulement en retournant le problème dans une perspective relativiste (les sociétés littératiennes sont organisées selon des configurations variables : code alphabétique vs idéographique, support matériel vs virtuel, littératie restreinte vs élargie, usages profanes de l'écrit vs usages sacrés, etc.), mais en prenant en compte le fait qu'il y a des degrés dans la littératie (et par conséquent dans l'oralité). Il est manifeste par exemple que le dictionnaire, le catalogue, le billet d'avion électronique, l'index, le roman, la table de multiplication, la carte, la réécriture, la relecture, la prise de notes, etc. présentent un très fort taux de littératie. Et inversement, " observer une minute de silence », «maudire » ou « chanter une berceuse » sont des rites fondamentalement oraux qui n'ont pas d'équivalents pragmatiques à l'écrit.

Mais, en fait, la plupart des usages langagiers présentent des taux de littératie convertibles à l'oral (le taux de change peut être très bas ou très élevé) ou se conjuguent avec des moments d'oralité. Ainsi, une lettre d'amour ne saurait être confondue avec une déclaration d'amour face to face, même si dans certains cas l'une peut se substituer ou préluder à l'autre. Et si dans les cérémonies républicaines de mariage la signature scelle officiellement le contrat, la présence physique des témoins est requise tout comme la profération rituelle d'un sonore et attendu « Oui ».

(3) G. Flaubert, Lettre à Louise Colet, 31 janvier 1852, Correspondance, II, éd. J. Bruneau, Paris, Gallimard / Pléiade, 1980, p. 42. 
Enfin, ce continuum dans les modes de communication est parfois à ce point incorporé que l'orateur peut « parler comme un livre », " ponctuer son discours », « mettre les points sur les i » et que l'écolier modèle qui a goûté à « la soupe à l'alphabet » et fait ses premiers apprentissages en lecture dans des abécédaires anthropomorphes, se doit de réciter « par cœur» 1'ordre alphabétique ou une fable de La Fontaine, sans en changer une virgule.

Ces hybridations sont peut-être plus nombreuses et plus constantes qu'une conception monolithique et canonique de la littératie ne le laisserait accroire. Y compris dans des textes littéraires attentifs à l'hétérogénéité de micro-gestes culturels où les dispositions d'une scripturalité épistolaire conventionnelle sont travaillées à même le texte par l'ethno-logique d'une oralité coutumière (contredon en nature, vocabulaire dialectal et surtout ré-ancrage initial de la déixis dans l'imaginaire nostalgique d'une relation de co-présence - et déni fugitif d'une douloureuse situation d'énonciation in absentia) :

"C'était l'époque où le père Rouault envoyait son ( sic) dinde, en souvenir de sa jambe remise. Le cadeau arrivait toujours avec une lettre. Emma coupa la corde qui la retenait au panier, et lut les lignes suivantes :

"Mes chers enfants,

J'espère que la présente vous trouvera en bonne santé et que celui-là vaudra bien les autres ; car il me semble un peu plus mollet, si j'ose dire, et plus massif. Mais, la prochaine fois, par changement, je vous donnerai un coq $[\ldots] . ”{ }^{(4)}$

\section{La raison graphique et ses concrétions}

Nous avons jusqu'ici essayé de complexifier les contours habituels du concept de littératie par une approche anthropologique dans une perspective de synchronie et de synergie culturelles (et pas seulement dans une perspective historique ou comparative classiques) et par une analyse des discours écrits, oraux et scriptoraux (si je peux risquer ce néologisme) en situation.

Une troisième façon de retravailler le concept de littératie conduit à élargir singulièrement la portée de l'arraisonnement graphique dans les sociétés littéracisées.

Si l'on définit - comme il est désormais admis assez communément depuis les travaux de J. Goody - qu'il y a des relations dialectiques fortes et constantes entre modes et moyens de communication, modes et moyens de pensée et modalités d'organisation sociale et culturelle (type d'Etat, de justice, d'économie, de religion, de sciences, d'arts, etc.), il paraît inconséquent de ne pas faire l'hypothèse, par exemple, que les technologies littératiennes de l'intellect ne coexisteraient pas avec une sorte de sémiotisation littératienne des technologies (et de leurs produits).

On a beaucoup parlé, jadis, à l'époque de «l'aventure sémiologique » (R. Barthes, 1985) et à propos de la ville par exemple, «d'énonciations piétonnières » et de « rhétoriques cheminatoires » ${ }^{(5)}$ et on a discuté pour savoir si effectivement

(4) G. Flaubert, Madame Bovary - Mours de province, Paris, Garnier Frères, 1971, pp. 175176.

(5) M. de Certeau, «Le parler des pas perdus », L'Invention du quotidien, Arts de faire, 1, Paris, UGE, 10/18, 1980, pp. 180-187. 
« la ville est structurée comme un langage » ${ }^{(6)}$. A vrai dire, les métaphores de la ville comme « texte » ou « discours » butaient régulièrement sur une difficulté majeure ou plutôt une généralisation abusive ou hâtive. L'organisation géométrique d'une grande ville moderne occidentale n'est pas simplement « un langage »; si la pensée urbanistique et la réalité urbaine (jardins, salles de concert, grands magasins, rues, cimetières, etc.) sont le produit d'une « civilisation du rectangle » ${ }^{(7)}$ c'est qu'elles matérialisent les structures ou mieux les infrastructures constitutives de la littératie, versant écrit du « langage».

Je m'explique. Souvenons-nous de la démonstration osée par P. Bourdieu visant à mettre à jour les affinités structurales et génétiques entre la culture scolastique des lettrés médiévaux et l'architecture gothique des bâtisseurs de cathédrale nourris de la Somme théologique de saint Thomas d'Aquin. Ouvrage écrit et ouvrage d'art sont de purs produits littératiens « au titre d'ensembles intelligibles composés selon des méthodes identiques, avec, entre autres traits, la séparation rigoureuse qui s'y établit entre les parties, la clarté expresse et explicite des hiérarchies formelles et la conciliation harmonieuse des contraires [...]. » ${ }^{(8)}$

Ces équivalences symboliques et pratiques seraient ainsi nécessairement actives « dans une société où la transmission de la culture est monopolisée par une école, les affinités profondes qui unissent les œuvres humaines (et bien sûr les conduites et les pensées) trouvant leur principe dans l'institution scolaire investie de la fonction de transmettre [...], ou plus exactement de produire des individus dotés de ce système de schèmes inconscients (ou profondément enfouis) qui constitue leur culture, ou mieux leur habitus [...]. " ${ }^{(9)}$ Ainsi, la pensée scolastique (système incorporé et intériorisé de dispositions cognitives et productives), pensée d'école et pensée structurée par une littératie subtilement organisée, ordonnée, planifiée, serait à la genèse culturelle de «l'homologie entre la croisée d'ogives et la brisure de l'écriture gothique. » ${ }^{(10)}$

Or, l'une des spécificités de l'écrit en général est de pouvoir spatialiser le langage sous forme de cartes orientées, de listes systématiques et de tableaux organisés de données textuelles, produits combinés d'une " habileté technique » et d'une « aptitude intellectuelle » ${ }^{(11)}$. En ce sens, la « tabularisation » et la « géométrisation » formatent le réel langagier et ces formats de pensée sont redoublés

(6) Voir par exemple les mises au point de F. Choay, L'Urbanisme, utopies et réalités, Paris, Points/Seuil, 1979 (1965), pp. 78-83 ou de B. Lamizet et P. Sanson, eds., Les Langages de la ville, Paris, Parenthèses, 1997.

(7) R. Barthes, "Rectangle », Comment vivre ensemble, éd. Cl. Coste, Paris, Seuil / Imec, 2002, pp. 158-161.

(8) P. Bourdieu, «Postface» à E. Panofsky, Architecture gothique et pensée scolastique, Paris, Minuit, 1967, pp. 135-167. V. Hugo, en ses fulgurances historiques et sémiotiques, comparait déjà « le livre imprimé » et « le livre architectural », « la bible de pierre »et « la bible de papier »; il admirait " la majesté visible de l'écriture de granit » des cathédrales et leurs "gigantesques alphabets formulés en colonnades » et il tenait l'œuvre de Shakespeare pour « la dernière cathédrale gothique. » (Notre-Dame de Paris, « Ceci tuera cela »). Mais alors que V. Hugo estime que «le livre va tuer l'édifice» nous pensons que l'édifice moderne est (structuré) comme un livre imprimé.

(9) Id., ibidem.

(10) Sur les relations (réciproques) entre la ville (classique et monarchique) et la raison (graphique et géométrique), « entre une forme spatiale, une structure sociale et un type mental», voir par exemple la contribution récente de P. Blanquart, «Ville royale, cartésianisme et techno-administration », Une Histoire de la ville, Paris, La Découverte / Essais, 1997, pp. 89-116.

(11) J. Goody, « Que contient une liste? », La Raison graphique, op. cit., p. 193. 
par la structure même du livre (codex) et par le format de la page (ce « champ » labouré par l'écriture selon les Anciens) dont les lignes sont ordonnées selon une régularité absolue et où « chaque lettre elle-même doit s'inscrire dans un carré sur un axe vertical ». Bref, le livre, ses pages, ses lignes, ses lettres s'emboîtent systématiquement pour construire un parfait " rectangle pensant ", dans une sorte de coalescence des structures logiques et des structures physiques de la littératie $^{(12)}$.

\section{A l'école de la littératie}

Ce primat de l'angle droit, de l'alignement, du parallélisme, de la symétrie, de la ligne droite et de l'intersection dans le monde littératien se retrouve dans l'apprentissage de l'écriture. En effet, avant d'être « mis dans la position de l'industriel, de l'urbaniste, ou du philosophe cartésien pour gérer l'espace propre et distinct $[. .$.$] de sa page blanche { }^{(13)}{ }^{2}$, l'enfant apprend à écrire, stricto sensu :

« L'écriture est un dessin [...]. Le maître marque un point sur le tableau noir [...]. Il pose un second point au-dessus du premier : "Voici un point haut et un point bas." - Les élèves apprennent ensuite à distinguer de la même façon la droite de la gauche; puis, tant à droite qu'à gauche, un point supérieur et un point inférieur [...]. Le maître trace alors une ligne entre un point supérieur et celui qui est verticalement au-dessous [...]. On grave dans la mémoire des enfants le sens du mot "vertical" en montrant des objets offrant dans leur construction des lignes verticales. Puis les enfants marquent sur leurs ardoises deux points, l'un au-dessus et l'autre au-dessous, et les réunissent par une ligne [...]. » ${ }^{(14)}$

C'est ainsi du moins que les enfants de l'Ecole de Jules Ferry apprenaient à tracer des figures géométriques régulières pour entrer en littératie... L / T / I et tous les croisements rectilignes et équidistants nécessaires.

Or, notre hypothèse pan-littératienne nous conduit à une sémiotique de la signification qui « lit» dans des lieux dépourvus de toute trace d'écrit l'infrastructure concrète de l'écriture, la matérialisation parfaite de ses constituants formels. Ainsi une piscine municipale, lieu par excellence où les écrits disparaissent à l'entrée du bassin, est-elle un pur produit de la pensée graphique, structurée exactement comme une page d'écolier...

Un début d'observation ethnographique me permet d'avancer que les piscines traditionnelles de centre ville sont souvent des lieux d'exercice où des corps très policés et très auto-disciplinés font en silence des lignes et comptent les longueurs en parcourant un espace quadrillé jusque dans le moindre détail - là tout n'est que formes géométriques et tracés rectilignes. Les très jeunes nageurs jouent de leur côté avec des bouées en forme de A ou de U. et apprennent à discipliner leurs corps. Le message (à peine) subliminal est qu'ici on est à l'école de la littératie et qu'on «baigne » non pas dans de l'écrit au sens académique du terme, mais, pour reprendre la très belle expression de M. Laparra, dans de «l'écrit chosifié "».

(12) Ces réflexions doivent beaucoup aux analyses très éclairantes de M. Melot, Livre, Paris, L'œil neuf éditions, 2006, pp. 17-136.

(13) M. de Certeau, «L'économie scripturaire », op. cit., p. 236.

(14) F. Buisson, Dictionnaire de pédagogie et d'instruction primaire, s.v. «lecture », $1^{\mathrm{re}}$ partie, tome second, Paris, Hachette, 1887. 
Cette anthropologie des structures formelles de la culture littératienne explique peut-être pourquoi dans les piscines nouvelles des quartiers périphériques vouées à de publics plus populaires l'organisation des espacements est asymétrique et discontinue, et, comme à dessein, leur utilisation labile, irrégulière, transversale - là tout n'est que lignes brisées et trajectoires fantaisistes de corps. A un premier espace « disciplinaire », linéairement réglé, hiérarchisé et articulé, expressément voué à un austère studium sous le regard d'un maître-nageur, est donc substitué un lieu multifonctionnel et très « vivant», dé-linéarisé et comme dé-régulé, qui appelle le ludus où s'entrecroisent le libre jeu des lignes et des corps. De toute façon, ce corps ludique et joyeusement expressif a ses déraisons que la raison (graphique) ne saurait encadrer trop longtemps (les règlements très « hygiénistes » et «puritains » affichés dans le hall d'entrée). Ainsi, la piscineexercice (scolaire) renforce-t-elle l'incorporation de la structuration graphique et éthique du sujet pendant que la piscine-jeu le divertit des contraintes insupportables d'une littératie trop envahissante.

\section{Coûts, goûts et dégoûts de la littératie}

En effet, l'acculturation performante à l'ordre littératien impose une exposition continue, diffuse et profuse, à ses lois. Or, les classes les plus éloignées des gratifications littératiennes (gratifications privées ou professionnelles) ont mis et continuent de mettre très souvent en avant le coût de ces apprentissages, ( « lire, ça me prend la tête »), sinon leur faible rendement personnel. Ou du moins, elles se comportent comme s'il en était ainsi en fuyant dans les temps libres festifs et collectifs le monde de la littératie, quitte à perdre pied dans les rudes apprentissages scripturaux. On comprend en effet que si la littéracisation est bien le processus complexe et englobant que nous avons décrit (capital littératien, coefficient de littératie, littératie infrastructurelle), les sujets les plus éloignés (mais aussi parfois aussi les plus impliqués...) puissent être tenus à distance et / ou se tenir à distance des formes les plus impérieuses de cette exigence symbolique et pratique.

C'est Coupeau, l'ouvrier zingueur analphabète et jouisseur qui refuse d'apprendre à lire car il « accuse la science de maigrir le monde » (E. Zola, L'Assommoir, 1877) ; c'est George Sand, «pauvre écrivassière attachée du matin au soir à son boulet et quelquefois rassasiée d'encre et de papier à la fin de sa journée au point de cacher au fond d'un tiroir, manuscrits, plumes d'oie, papier Whatman, encre [...] et tout l'odieux attirail de [son] métier pour n'en pas même supporter la vue. " ${ }^{(15)}$ Tout le monde n'a pas un (notre ?) inaltérable habitus littératien: «Je me demande sérieusement s'il est possible qu'il y ait des êtres sur la terre s'occupant à autre chose qu'à aligner des phrases et à chercher des adjectifs. " ${ }^{(16)}$ Beaucoup trouvent en effet plus de plaisirs à faire des ronds dans l'o...

(15) G. Sand, Lettre à Emile Paultre, Nohant, 25 août 1832, Correspondance, Paris, Garnier, II, 1966 , p. 145.

(16) G. Flaubert, Par les champs et par les grèves, éd. P-L. Rey, Paris, Pocket, 2002, p. 151. 